\title{
Tissue-type plasminogen activator promotes murine myofibroblast activation through LDL receptor-related protein 1-mediated integrin signaling
}

\author{
Kebin Hu, Chuanyue Wu, Wendy M. Mars, and Youhua Liu \\ Department of Pathology, University of Pittsburgh School of Medicine, Pittsburgh, Pennsylvania, USA.
}

\begin{abstract}
The activation of interstitial fibroblasts to become $\alpha$-SMA-positive myofibroblasts is an essential step in the evolution of chronic kidney fibrosis, as myofibroblasts are responsible for the production and deposition of the ECM components that are a hallmark of the disease. Here we describe a signaling pathway that leads to this activation. Tissue-type plasminogen activator (tPA) promoted TGF- $\beta 1$-mediated $\alpha$-SMA and type I collagen expression in rat kidney interstitial fibroblasts. This fibrogenic effect was independent of its protease activity but required its membrane receptor, the LDL receptor-related protein 1 (LRP-1). In rat kidney fibroblasts, tPA induced rapid LRP-1 tyrosine phosphorylation and enhanced $\beta 1$ integrin recruitment by facilitating the LRP-1/ $\beta 1$ integrin complex formation. Blockade or knockdown of $\beta 1$ integrin abolished type I collagen and $\alpha$-SMA expression. Furthermore, inhibition of the integrin-linked kinase (ILK), a downstream effector of $\beta 1$ integrin, or disruption of $\beta 1$ integrin/ILK engagement, abrogated the tPA action, whereas ectopic expression of ILK mimicked tPA in promoting myofibroblast activation. In murine renal interstitium after obstructive injury, tPA and $\alpha$-SMA colocalized with LRP-1, and tPA deficiency reduced LRP-1/ $\beta 1$ integrin interaction and myofibroblast activation. These findings show that tPA induces LRP-1 tyrosine phosphorylation, which in turn facilitates the LRP-1-mediated recruitment of $\beta 1$ integrin and downstream ILK signaling, thereby leading to myofibroblast activation. This study implicates tPA as a fibrogenic cytokine that promotes the progression of kidney fibrosis.
\end{abstract}

\section{Introduction}

Interstitial fibrosis is considered the common final outcome of a wide variety of chronic kidney diseases (CKDs), regardless of the initial causes $(1,2)$. In a simplistic view, renal interstitial fibrosis is a deteriorating process characterized by massive fibroblast/ myofibroblast activation and excessive production and deposition of ECM, which leads to the destruction and collapse of renal parenchyma and progressive loss of kidney function. Because the $\alpha$-SMA-positive myofibroblasts are the principal effector cells that are responsible for ECM overproduction in the fibrotic kidney, their activation is regarded as a key event in the pathogenesis of chronic renal fibrosis $(3,4)$. Indeed, a large body of evidence shows that the extent of myofibroblast activation is closely correlated with the prognosis of CKD in both animal models and patients (5-8). Although myofibroblasts may come from different sources such as tubular epithelium through an epithelialmesenchymal transition $(9,10)$, interstitial fibroblast activation arguably remains the major pathway leading to the generation of the matrix-producing myofibroblasts. However, the regulation and mechanism of myofibroblast activation in pathologic conditions remain poorly understood.

Nonstandard abbreviations used: CKD, chronic kidney disease; ILK, integrin-linked kinase; LRP-1, LDL receptor-related protein 1; NRK-49F, normal rat kidney interstitial fibroblast; RAP, receptor-associated protein; tPA, tissue-type plasminogen activator; UUO, unilateral ureteral obstruction.

Conflict of interest: The authors have declared that no conflict of interest exists. Citation for this article: J. Clin. Invest. 117:3821-3832 (2007). doi:10.1172/JCI32301.
Earlier studies from this laboratory demonstrate that genetic ablation of tissue-type plasminogen activator (tPA) protects the kidney from the development of interstitial fibrosis in obstructive nephropathy, as shown by a reduced myofibroblast accumulation and matrix deposition (11). This implicates tPA as an important player in the regulation of myofibroblast activation in vivo. tPA, a member of the serine protease family, plays a pivotal role in the homeostasis of blood coagulation and fibrinolysis. In the kidney, the main function of tPA is to convert plasminogen into biologically active plasmin, which in turn participates in the regulation of matrix homeostasis by its proteolytic potential. However, growing evidence suggests that tPA elicits many biological activities by a mechanism independent of its protease activity (12-14). We have recently demonstrated that tPA is able to upregulate MMP-9 gene expression, which is mediated by the LDL receptor-related protein 1 (LRP-1) (15). These observations establish that tPA acts as a cytokine that is capable of transducing its signal across the plasma membrane into the nucleus to control specific gene expression. In this context, it is conceivable to speculate that tPA, through its cytokine activity, may activate its membrane receptor, trigger a cascade of signal transduction events, and finally modulate myofibroblast activation.

Although there is no dedicated, so-called tPA receptor thus far, accumulating evidence suggests that LRP-1, a member of the LDL receptor family, may serve as tPA functional receptor and mediates its signal transduction (15). Mature LRP-1 consists of an external $515-\mathrm{kDa} \alpha$ subunit and an $85-\mathrm{kDa} \beta$ subunit that contains a transmembrane segment and cytoplasmic tail with numer- 


$$
\text { A }
$$$$
\frac{12 \mathrm{~h}}{-+-+} \frac{24 \mathrm{~h}}{-+-+\mathrm{tPA}}
$$$$
--++-++ \text { TGF-B1 }
$$

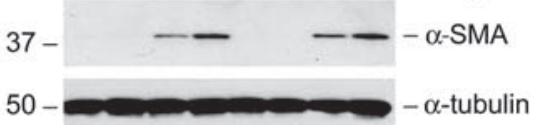

D
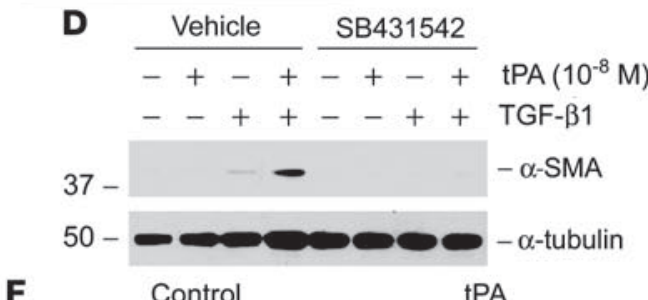

$\mathbf{F}$
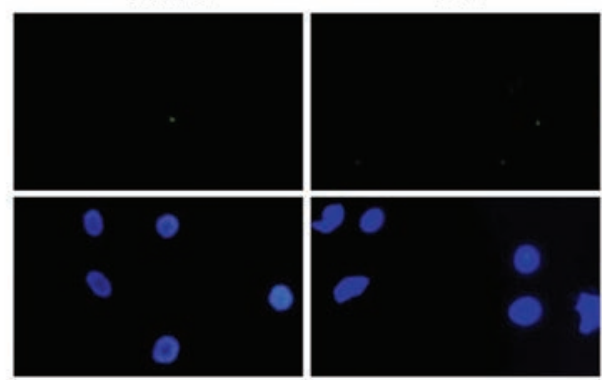

B
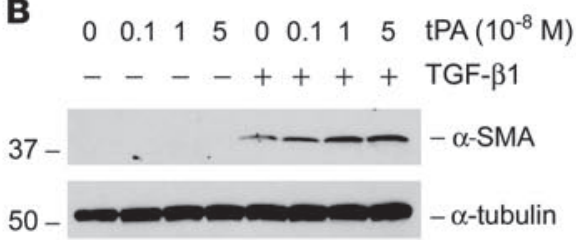

E

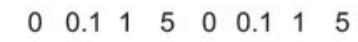

-+-++++ TGF- $\beta 1$

$0.3 \mathrm{~kb}$

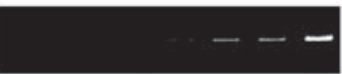

- Collagen 1

$0.3 \mathrm{~kb}$

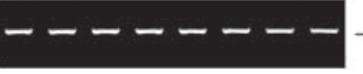

$-\beta$-actin

$\mathrm{A}\left(\mathrm{X} 10^{-8} \mathrm{M}\right)$
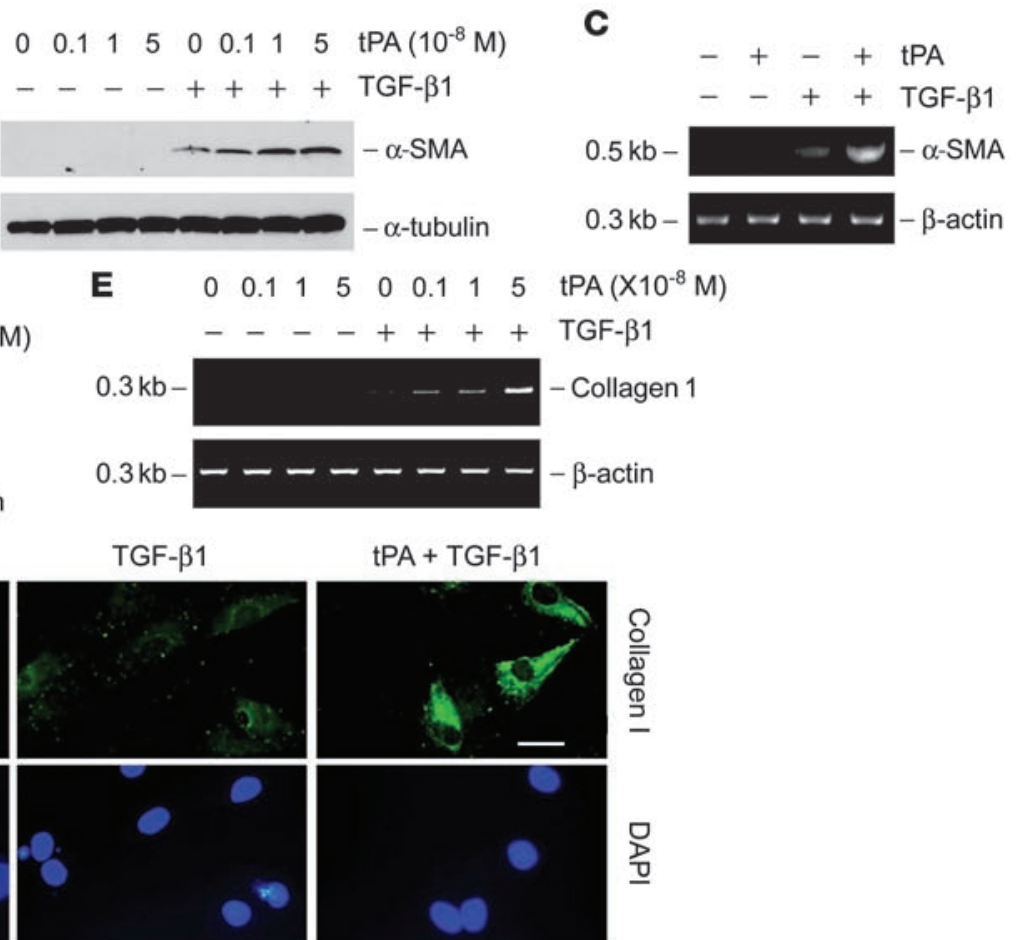

$\mathrm{tPA}+\mathrm{TGF}-\beta 1$

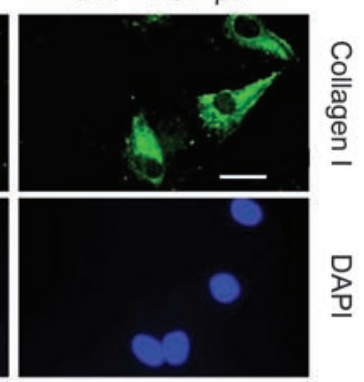

Figure 1

tPA promotes TGF- $\beta 1$-mediated myofibroblast activation from renal interstitial fibroblasts. NRK-49F cells were treated with $10^{-8} \mathrm{M}$ tPA and/or $0.5 \mathrm{ng} / \mathrm{ml} \mathrm{TGF}-\beta 1$ for 24 hours, unless indicated otherwise. (A and B) Western blot analyses demonstrated that tPA synergistically promoted the TGF- $\beta 1$-induced $\alpha$-SMA expression in a time- (A) and dose-dependent manner (B) in NRK-49F cells. (C) RT-PCR shows that tPA promoted the $\alpha$-SMA mRNA expression induced by TGF- $\beta 1$. (D) Blockade of TGF- $\beta$ signaling abolished the synergistic effect of tPA. NRK-49F cells were treated with $10^{-8} \mathrm{M} \mathrm{tPA}$ and/or $0.5 \mathrm{ng} / \mathrm{ml}$ TGF- $\beta 1$ in the absence or presence of the TGF- $\beta$ type I receptor inhibitor SB431542 (10 $\left.\mu \mathrm{M}\right)$. (E) RT-PCR shows that tPA promoted the TGF- $\beta 1$-mediated mRNA induction of type I collagen. (F) Immunofluorescence staining shows that tPA promoted TGF- $\beta 1$-induced type I collagen expression in NRK-49F cells. Scale bar: $20 \mu \mathrm{m}$.

ous tyrosine residues $(16,17)$. Generally considered a scavenger receptor, the unique molecular structure of LRP-1 endows it the potential to mediate the cellular signaling (18). It is unknown, however, whether tPA/LRP-1 plays a role in the regulation of myofibroblast activation; and if so, what is the downstream effector that transmits its signal?

In this study, we demonstrate that tPA promotes TGF- $\beta 1$-mediated renal myofibroblast activation and ECM production. The fibrogenic action of tPA is apparently mediated by its activation of LRP-1, which triggers LRP-1 tyrosine phosphorylation and facilitates the LRP-1-mediated recruitment of $\beta 1$ integrin, subsequently activating the downstream integrin-linked kinase (ILK) signaling. Our results have defined what we believe to be a novel pathway in which tPA modulates myofibroblast activation through a cascade of signaling events.

\section{Results}

tPA synergistically promotes TGF- $\beta 1$-triggered renal interstitial myofibroblast activation. We first investigated whether $\mathrm{tPA}$ modulates the myofibroblastic activation from interstitial fibroblasts, a key event in generating the matrix-producing effector cells in renal fibrogenesis. Using normal rat kidney interstitial fibroblast (NRK-49F) cells as a model system, we found that tPA alone did not induce de novo expression of $\alpha$-SMA, the molecular hallmark of myofibroblast activation (Figure 1A) (8). However, simultaneous incubation of NRK-49F cells with tPA substantially promoted TGF- $\beta 1$-mediated $\alpha$-SMA expression in a time-dependent manner (Figure $1 \mathrm{~A}$ ), suggesting that $\mathrm{tPA}$ can synergistically potentiate the fibrogenic action of TGF- $\beta 1$. The effect of tPA on TGF- $\beta 1-$ mediated $\alpha$-SMA expression was also dose dependent (Figure 1B). Similarly, tPA markedly promoted TGF- $\beta 1$-mediated $\alpha$-SMA mRNA expression in NRK-49F cells, whereas tPA alone had little effect on $\alpha$-SMA mRNA expression (Figure 1C). Notably, a TGF- $\beta$ type I receptor inhibitor, SB431542, was able to abolish the synergistic effect of tPA on $\alpha$-SMA expression (Figure 1D), suggesting its dependency on TGF- $\beta$ signaling. As myofibroblast activation is accompanied by the overproduction of interstitial matrix components that leads to tissue scarring, we next examined the effect of tPA on ECM expression in NRK-49F cells. As shown in Figure 1, tPA synergistically promoted the type I collagen mRNA and protein expression induced by TGF- $\beta 1$ in NRK-49F cells (Figure $1, \mathrm{E}$ and F). tPA also enhanced the TGF- $\beta 1$-induced fibronectin mRNA expression (data not shown). Together, it appears clear that tPA is a profibrotic factor that synergistically potentiates the TGF- $\beta 1$-mediated myofibroblast activation and matrix production.

To test whether tPA is similarly important in the activation of fibroblasts beyond the kidney, we examined the effects of tPA on the myofibroblast activation in nonrenal fibroblasts. As shown in Supplemental Figure 1 (supplemental material available online with this article; doi:10.1172/JCI32301DS1), tPA also synergisti- 


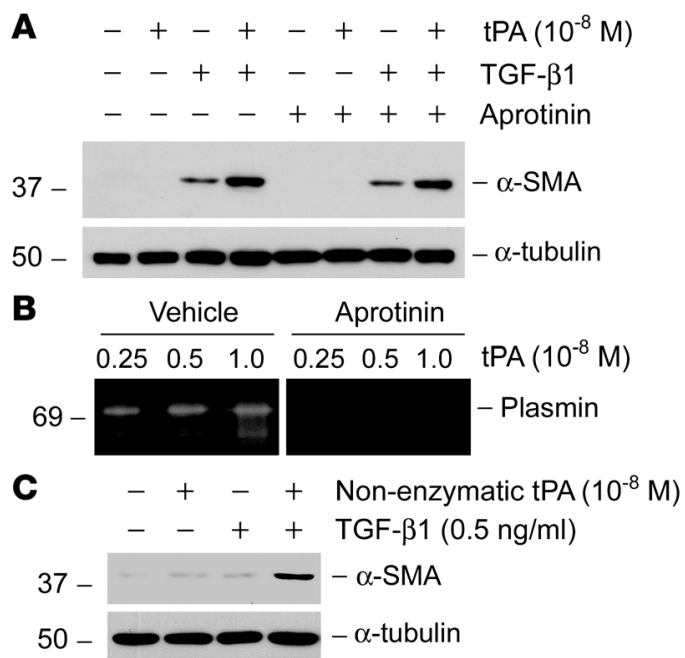

cally promoted TGF- $\beta 1$-mediated $\alpha$-SMA expression in human lung fibroblasts (MRC-5) and primary rat hepatic stellate cells. Therefore, these findings appear to have broad implications and may be of general importance in organ fibrogenesis.

\section{Figure 2}

tPA promotes myofibroblast activation by a mechanism independent of its protease activity. (A) Plasmin inhibitor aprotinin $(50 \mathrm{U} / \mathrm{ml})$ did not affect the synergistic effect of tPA with TGF- $\beta 1(0.5 \mathrm{ng} / \mathrm{ml})$ on $\alpha$-SMA induction in NRK-49F cells. (B) Zymographic analysis demonstrates that aprotinin $(50 \mathrm{U} / \mathrm{ml})$ was able to block the proteolytic activity of plasmin. (C) Non-enzymatic mutant tPA retained the ability to synergistically promote $\alpha$-SMA expression induced by TGF- $\beta 1$. NRK- $49 \mathrm{~F}$ cells were incubated with mouse recombinant non-enzymatic IPA in the absence or presence of TGF- $\beta 1$, as indicated.

The fibrogenic effect of $t P A$ is independent of its protease activity. Because tPA is a serine protease that converts plasminogen into biologically active plasmin and is also implicated in the posttranslational activation of several growth factors including TGF- $\beta 1$ (19), the possibility exists that tPA may promote myofibroblast activation through a circumlocutory mechanism that depends on its protease activity. To test this, 2 approaches were employed. First, we explored whether inhibition of plasmin, the major product of tPA proteolytic activity, affects its ability to promote myofibroblast activation. As shown in Figure 2A, pretreatment of NRK-49F cells with aprotinin, a potent plasmin inhibitor, did not affect the synergistic effect of tPA on $\alpha$-SMA induction, indicating that tPA exerts its fibrogenic action
A

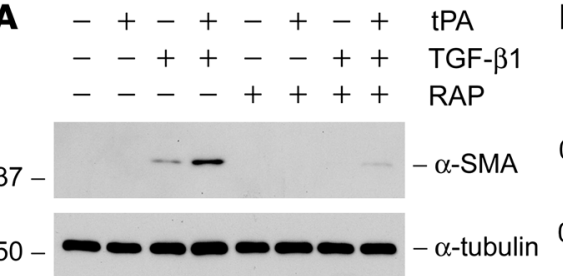

D

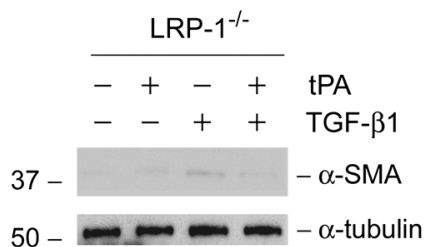

G

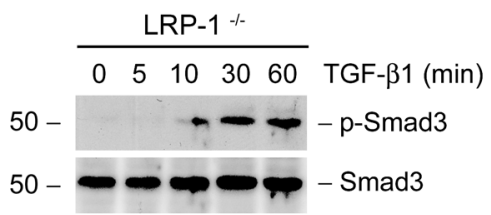

B

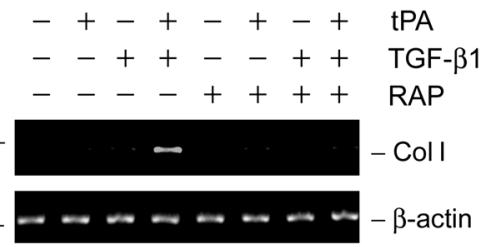

E

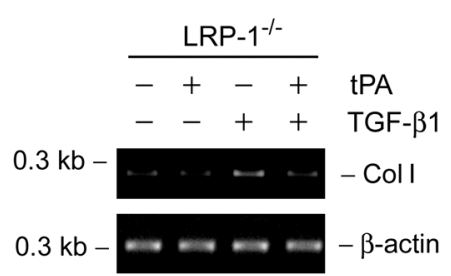

H

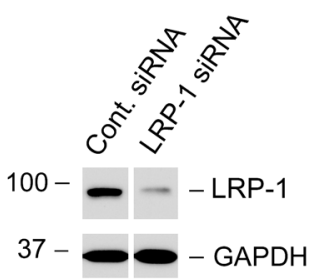

I
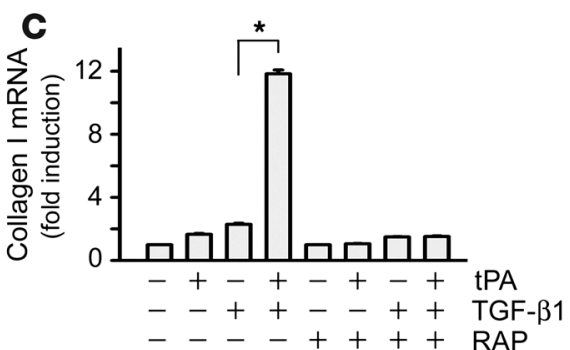

F
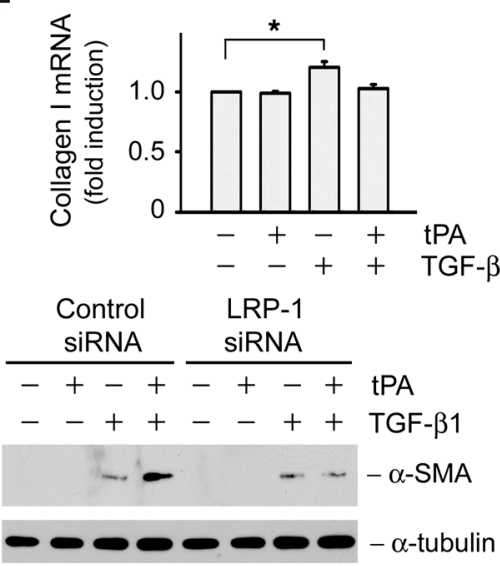

Figure 3

LRP-1 receptor is required for tPA to elicit its fibrogenic action. (A-C) The RAP, an antagonist of LRP-1, abolished the effect of tPA on myofibroblast activation. The synergistic effect of tPA on $\alpha$-SMA (A) and type I collagen (B and $\mathbf{C})$ induction was abrogated in the presence of RAP $(0.5 \mu \mathrm{M})$ in NRK-49F cells. (D-F) Ablation of LRP-1 abolished the effect of tPA on myofibroblast activation. tPA failed to promote TGF- $\beta 1-$ induced $\alpha$-SMA (D) and type I collagen (E and F) expression in LRP-1-deficient PEA-13 fibroblasts. tPA, $10^{-8} \mathrm{M} ;$ TGF- $\beta 1,0.5 \mathrm{ng} / \mathrm{ml}$. Quantitative presentation of type I collagen mRNA levels in different groups is given in $\mathbf{C}$ and $\mathbf{F}$. ${ }^{*} P<0.05$. (G) TGF- $\beta 1$ induced Smad3 phosphorylation and activation in LRP-1-deficient PEA-13 cells. Cell lysates were prepared at different time points after stimulation with TGF- $\beta 1$ (2 ng/ml) and immunoblotted with antibodies against phospho-specific and total Smad3. (H and I) Knockdown of LRP-1 in NRK-49F cells abolished the synergistic effect of tPA. (H) Western blot analysis demonstrated the downregulation of LRP-1 in NRK-49F cells after transfection of the LRP-1-specific siRNA. The lanes were run on the same gel but were noncontiguous. (I) Downregulation of LRP-1 in NRK-49F cells abolished the $\alpha$-SMA expression induced by tPA $\left(10^{-8} \mathrm{M}\right)$, but not TGF- $\beta 1(0.5 \mathrm{ng} / \mathrm{ml})$. 
A

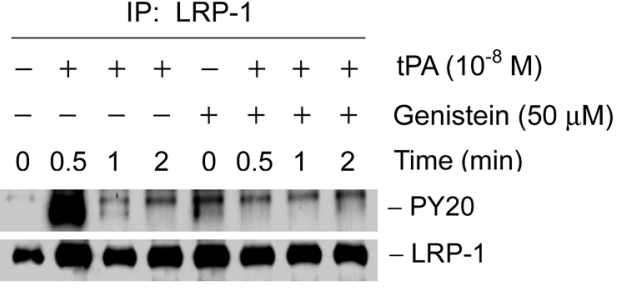

75

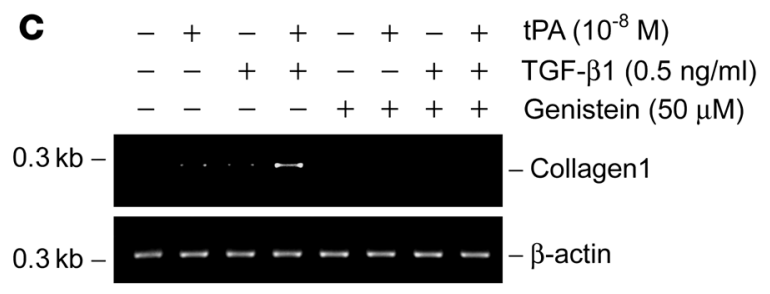

E

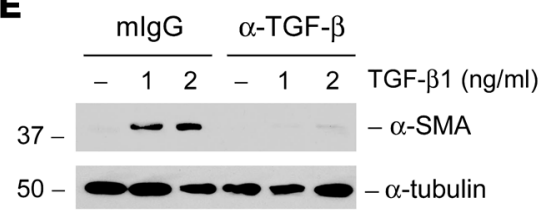

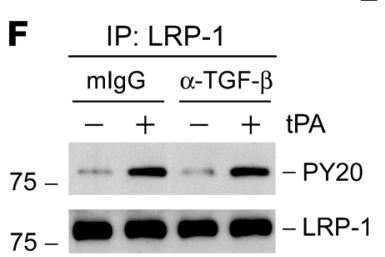
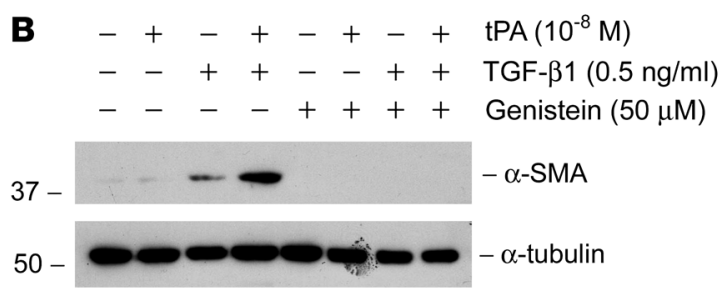

D

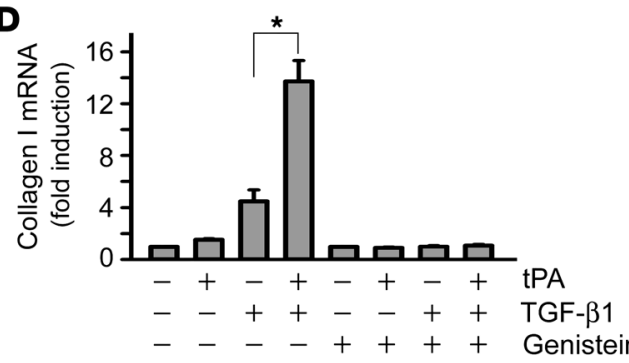

G

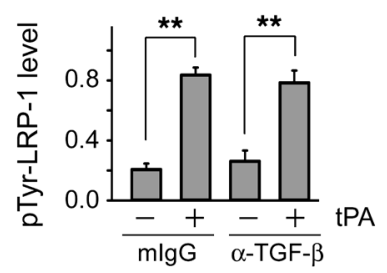

Figure 4

Tyrosine phosphorylation of LRP-1 is required for mediating the fibrogenic action of TPA and is independent of TGF- $\beta$ signaling. (A) IPA induced a rapid tyrosine phosphorylation of LRP-1 on its $\beta$ subunit, and genistein abolished tPA-triggered LRP-1 phosphorylation. Cell lysates after various treatments were immunoprecipitated with anti-LRP-1 antibody, followed by immunoblotting with anti-phosphotyrosine antibody (PY20). (B-D) Inhibition of tyrosine phosphorylation of LRP-1 by genistein abolished the effects of TPA on $\alpha$-SMA (B) and type I collagen (C and D) induction. Quantitative determination of type I collagen mRNA levels in different groups is presented in $\mathbf{D}$. ${ }^{*} P<0.05$. (E) Monoclonal pan-specific TGF- $\beta$-neutralizing antibody ( $\alpha$-TGF- $\beta)(25 \mu \mathrm{g} / \mathrm{ml})$ blocked the $\alpha$-SMA expression induced by TGF- $\beta 1$ in NRK-49F cells. Same amount of normal mouse $\operatorname{lgG}(\mathrm{mlgG})$ was used as negative controls. ( $F$ and $\mathbf{G}$ ) Blockade of TGF- $\beta$ signaling with neutralizing antibody did not affect the tPA-induced LRP-1 tyrosine phosphorylation. Representative western blot $(\mathbf{F})$ and graphic presentation of the relative phosphorylated LRP-1 levels $(\mathbf{G})$ are presented. ${ }^{* *} P<0.01$.

by a mechanism independent of plasmin generation. Of note, aprotinin at the concentration used was capable of blocking plasmin activity in a zymographic assay (Figure 2B). Next, we directly examined whether the protease activity of tPA is required for promoting myofibroblast activation by using nonenzymatic mutant tPA. Recombinant nonenzymatic tPA has the serine within the active site of the enzyme mutated to alanine, which renders it catalytically inactive but retains its binding properties $(20,21)$. As shown in Figure $2 \mathrm{C}$, nonenzymatic tPA retained its ability to synergistically promote $\alpha$-SMA expression induced by TGF- $\beta 1$ in NRK- $49 \mathrm{~F}$ cells. These results indicate that the fibrogenic effect of tPA is mediated by a mechanism independent of its protease activity.

$L R P-1$ receptor mediates the fibrogenic action of tPA. As LRP- 1 is a functional tPA receptor that is capable of transmitting tPA signaling across the plasma membrane, we next investigated whether the fibrogenic effect of tPA on myofibroblast activation is mediated by LRP-1. As shown in Figure 3A, when NRK-49F cells were pretreated with the receptor-associated protein (RAP), a potent antagonist known to compete for the binding with all LRP-1 ligands (22), tPA failed to promote $\alpha$-SMA expression. Likewise, RAP also completely abolished the ability of tPA to synergistically promote TGF- $\beta 1$-mediated type I collagen expression in cells (Figure 3, B and $\mathrm{C}$ ). These results underline that the fibrogenic effect of tPA is dependent on its binding to LRP-1. Furthermore, in LRP-1-defi- cient mouse embryo fibroblasts (PEA-13), tPA could not potentiate the TGF- $\beta 1$-mediated $\alpha$-SMA and type I collagen expression (Figure 3, D-F). Of note, TGF- $\beta 1$ was still able to induce $\alpha$-SMA and type I collagen expression in PEA-13 cells at a magnitude similar to that in NRK-49F cells. In addition, TGF- $\beta 1$ was able to stimulate Smad3 phosphorylation and activation in PEA-13 cells (Figure 3G), suggesting that knockout of LRP-1 only affects the fibrogenic action elicited by tPA but not TGF- $\beta 1$.

To further ascertain the involvement of LRP- 1 in mediating the fibrogenic action of tPA, we knocked down LRP-1 expression in NRK-49F cells using a small interfering RNA (siRNA) approach. As shown in Figure 3H, LRP-1-specific siRNA caused a substantial reduction of LRP-1 in NRK-49F cells, comparing with control siRNA. Downregulation of LRP-1 was able to abolish the synergistic effects of tPA on $\alpha$-SMA induction, but did not affect the basal $\alpha$-SMA expression induced by TGF- $\beta 1$ (Figure 3I). Together, these results indicate that LRP-1 receptor is required for tPA to exert its fibrogenic action in renal interstitial fibroblasts.

Tyrosine phosphorylation of LRP-1 is required for mediating $t P A$ action. We have previously shown that the binding of tPA triggers a rapid phosphorylation of LRP- 1 on the tyrosine residues of the $\beta$ subunit, which leads to sequential events of signal transduction in NRK-49F cells. This finding prompted us to investigate whether the tyrosine phosphorylation of LRP- $1 \beta$ subunit is important in mediating tPA 
A

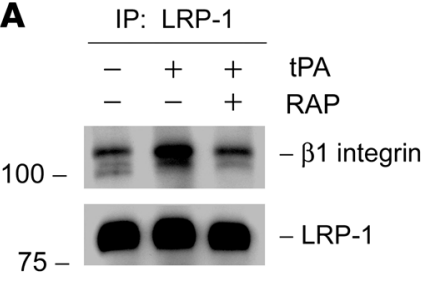

$\mathbf{E}$

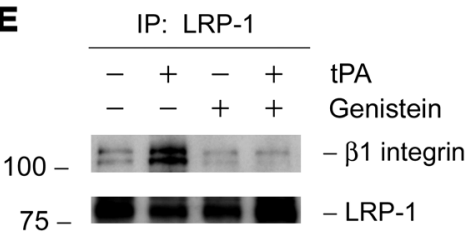

B

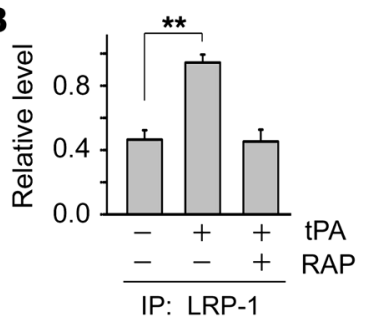

$\mathbf{F}$
C

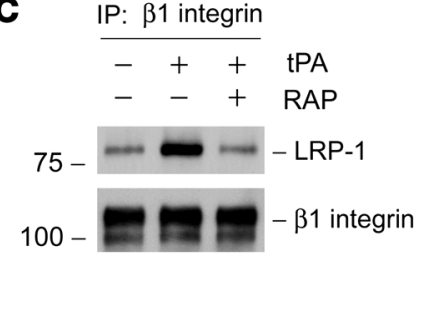

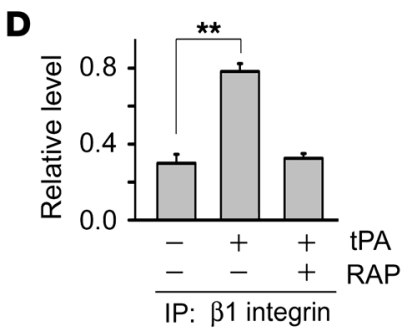

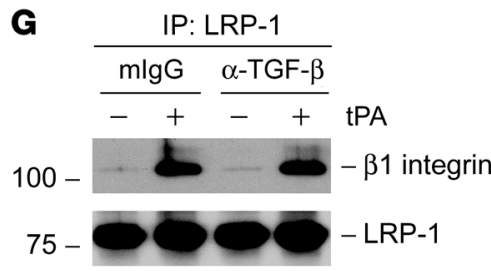

Figure 5

tPA promotes LRP-1 and $\beta 1$ integrin interaction and facilitates LRP-1-mediated recruitment of $\beta 1$ integrin. (A-D) Coimmunoprecipitation shows that IPA promoted the physical interaction of LRP-1 and $\beta 1$ integrin, while RAP abolished such interaction in NRK-49F cells. Cell lysates were precipitated with anti-LRP-1, followed by immunoblotting with antibodies against $\beta 1$ integrin or LRP-1, respectively (A and $\mathbf{B})$. In reciprocal experiments, cell lysates were first precipitated with anti- $\beta 1$ integrin, followed by blotting with antibodies against LRP-1 or $\beta 1$ integrin (C and D). Representative western blots ( $\mathbf{A}$ and $\mathbf{C})$ and graphic representations of the relative levels of the LRP-1/ $\beta 1$ integrin complexes (B and $\mathbf{D})$ are presented. ${ }^{* \star} P<0.01$. (E) Inhibition of LRP-1 tyrosine phosphorylation by genistein $(50 \mu \mathrm{M})$ abolished the tPA ability to promote LRP-1/31 integrin interaction. (F) Treatments of NRK-49F cells with tPA for 2 hours had no effect on the abundance of total (top panel) or the cell membrane surface $\beta 1$ integrin (bottom panel). (G) Blockade of TGF- $\beta$ signaling with neutralizing antibody $(25 \mu \mathrm{g} / \mathrm{ml})$ did not affect the tPA-induced LRP- $1 / \beta 1$ integrin complex formation. Same amount of normal mouse $\operatorname{lgG}(\mathrm{mlgG})$ was used as a negative control.

action. Therefore, NRK-49F cells were pretreated with genistein, a potent tyrosine kinase inhibitor, to abrogate LRP-1 tyrosine phosphorylation. As shown in Figure 4A, tPA induced a rapid, robust, and transient phosphorylation of LRP-1 on its tyrosine residues in the $\beta$ subunit, and genistein completely abolished the tPA-induced LRP-1 phosphorylation. It appeared that tyrosine phosphorylation and activation of LRP-1 receptor was critical for mediating the fibrogenic action of $\mathrm{PAA}$, as genistein abolished the synergistic induction of $\alpha$-SMA and type I collagen (Figure 4, B-D).

LRP- 1 is also known as TGF- $\beta$ receptor $V(T \beta R-V)$, which serves as a co-receptor for TGF- $\beta$ and mediates TGF- $\beta$-induced growth inhibition in certain cell types $(23,24)$. To test whether TGF- $\beta 1$ also modulates tPA-mediated LRP-1 signaling, we examined LRP-1 phosphorylation after depletion of endogenous TGF- $\beta$ in NRK-49F cells. As shown in Figure 4E, a pan-specific TGF- $\beta$ neutralizing antibody was able to abolish the $\alpha$-SMA induction by TGF- $\beta 1$. However, incubation of NRK-49F cells with this antibody did not affect LRP-1 tyrosine phosphorylation induced by tPA (Figure 4, F and $G$ ). Hence tPA appears to directly induce LRP-1 tyrosine phosphorylation by a TGF- $\beta$-independent mechanism.

tPA facilitates the LRP-1-mediated recruitment of $\beta 1$ integrin. In view of the importance of integrin signaling in renal fibrosis, we speculated that $\mathrm{PA}$ may promote myofibroblast activation through regulating the integrin-mediated signal transduction. To explore any potential connection of tPA and integrin signaling, we investigated the possibility of an interaction between LRP- 1 and $\beta 1$ integrin by coimmunoprecipitation. As shown in Figure 5A, under normal unstimulated conditions, $\beta 1$ integrin could be detected in the immunocomplexes precipitated by anti-LRP-1 antibody, suggesting that LRP-1 constitutively interacts with $\beta 1$ integrin in the plasma membrane of renal interstitial fibroblasts. Interestingly, tPA apparently enhanced the complex formation between LRP- 1 and $\beta 1$ integrin, while LRP- 1 antagonist RAP abolished such interaction (Figure 5, A and B). In the reciprocal experiments, LRP-1 was also detectable in the complexes precipitated by anti- $\beta 1$ integrin antibody (Figure $5 \mathrm{C}$ ). Similarly, tPA facilitated the complex formation of $\beta 1$ integrin/ LRP-1 (Figure 5, C and D). We found that the tyrosine phosphorylation of LRP- 1 was required for it to interact with $\beta 1$ integrin, as genistein inhibited tPA-mediated LRP-1/ $\beta 1$ integrin interaction (Figure 5E). Short-term treatment with tPA, RAP, and genistein appeared not to affect the $\beta 1$ integrin abundance in NRK-49F cells, as demonstrated by western blot analysis of whole-cell lysates (Figure 5F). In addition, neither tPA nor genistein affected the $\beta 1$ integrin abundance on the surface of cell membrane. In an assay to detect cell membrane surface protein after biotinylation, a virtually equal amount of $\beta 1$ integrin was detected in the complexes precipitated by the streptavidin-conjugated agarose bead (Figure $5 \mathrm{~F}$ ). Furthermore, depletion of TGF- $\beta$ by neutralizing antibody did not affect the tPA-induced interaction between LRP- 1 and $\beta 1$ integrin (Figure 5G). These results suggest that an increased LRP- $1 / \beta 1$ integrin complex formation after tPA stimulation is primarily caused by the LRP-1-mediated recruitment of preexisting $\beta 1$ integrin and is independent of TGF- $\beta$ signaling.

Blockade of $\beta 1$ integrin signaling abolishes the $t P A$-mediated myofibroblast activation. Increased LRP-1-induced recruitment of $\beta 1$ integrin after tPA stimulation may potentially lead to their clustering in cell membrane, thereby activating integrin signaling. To test this hypothesis, we assessed the functional consequence of inhibiting $\beta 1$ integrin signaling by a neutralizing antibody. As demonstrated in Figure 6A, $\beta 1$ integrin antibody significantly blocked tPA-mediated myofibroblast activation, as evidenced by a reduced $\alpha$-SMA induction. Similarly, blockade of $\beta 1$ integrin signaling also abolished the synergistic effect of tPA and TGF- $\beta 1$ on type I collagen mRNA expression in NRK-49F cells (Figure 6, 
A

$$
\begin{aligned}
& -+-+-+-+-+-+\operatorname{tPA}\left(10^{-8} \mathrm{M}\right) \\
& --++--++--++ \text { TGF- } \beta 1 \\
& --_{-}++++-{ }_{-}-{ }_{-} \text {mouse } \operatorname{lgG} \\
& -{ }_{-}-{ }_{-}-{ }_{-}++++ \text {anti- } \beta 1 \text { integrin } \\
& -\quad--\alpha-S M A
\end{aligned}
$$

B

$$
\begin{aligned}
& -+-+-+-+-+-+\operatorname{tPA}\left(10^{-8} \mathrm{M}\right) \\
& --++--++--++ \text { TGF- } \beta 1 \\
& --_{-}++++{ }_{-}-c_{-} \text {mouse lgG }
\end{aligned}
$$

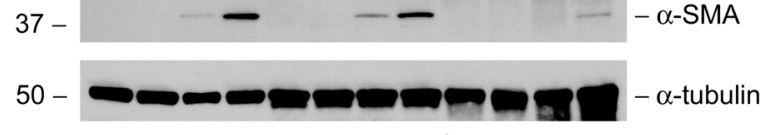$$
-c_{-}-c_{-}-+++
$$

anti- $\beta 1$ integrin

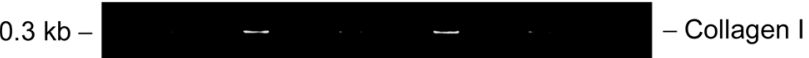

$0.3 \mathrm{~kb}$

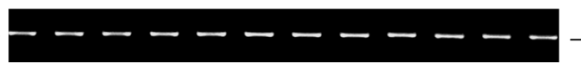
$-\beta$-actin

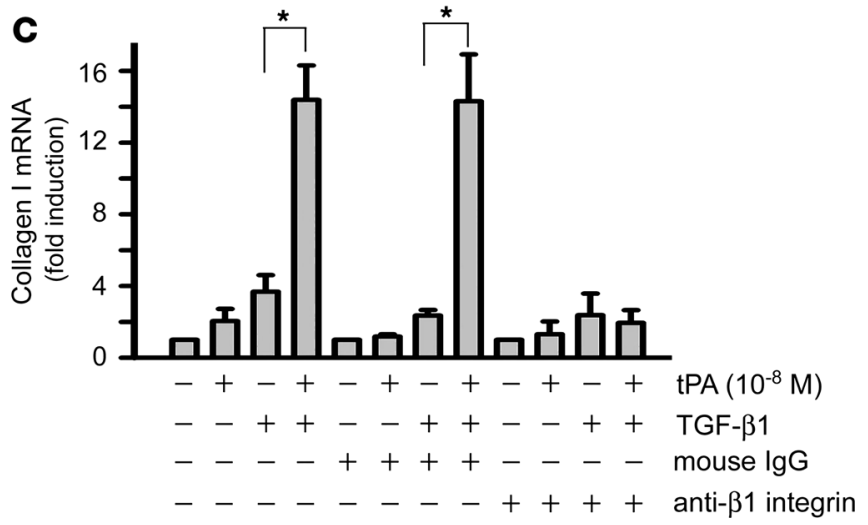

D
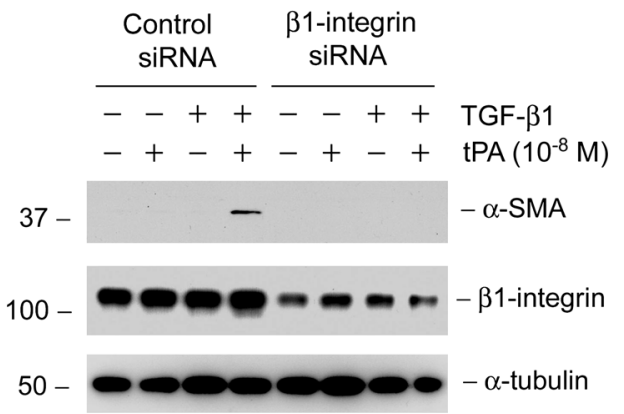

Figure 6

Either blockade of $\beta 1$ integrin signaling with neutralizing antibody or knockdown of its expression abolishes the tPA-mediated induction of myofibroblast activation. (A-C) NRK-49F cells were pretreated with mouse lgG or $\beta 1$ integrin antibody (12.5 $\mu \mathrm{g} / \mathrm{ml})$ for 1 hour, followed by treatment with TPA in the absence or presence of TGF- $\beta 1$ for 24 hours. Blockade of $\beta 1$ integrin signaling by neutralizing antibody eradicated the tPA-mediated induction of $\alpha$-SMA (A) and type I collagen (B and C) in NRK-49F cells. Quantitative determination of type I collagen mRNA levels in different groups is presented in $\mathbf{C}$. ${ }^{*} P<0.05$. (D) Knockdown of $\beta 1$ integrin also abolished the tPA-mediated induction of myofibroblast activation. NRK-49F cells were transfected with $\beta 1$ integrin-specific siRNA or control siRNA, followed by incubating with tPA in the absence or presence of TGF- $\beta 1$. Knockdown of $\beta 1$ integrin expression was confirmed by western blot analysis.

B and C). As a negative control, normal mouse IgG had no effects on tPA promotion of myofibroblast activation and matrix expression (Figure 6, A-C).

To confirm further the involvement of $\beta 1$ integrin in mediating tPA action, we sought to investigate the effect of downregulation of $\beta 1$ integrin on myofibroblast activation. Therefore, NRK-49F cells were transfected with $\beta 1$ integrin-specific siRNA or control siRNA, followed by incubating with tPA in the absence or presence of TGF- $\beta 1$. As shown in Figure $6 \mathrm{D}$, knockdown of $\beta 1$ integrin expression was confirmed by western blot analysis. We found that downregulation of $\beta 1$ integrin also abolished the tPA-mediated induction of myofibroblast activation (Figure 6D). Hence $\beta 1$ integrin signaling plays a critical role in mediating the fibrogenic action of tPA.

$I L K$, a downstream effector kinase of $\beta 1$ integrin, is required for mediating the fibrogenic action of $t P A$. ILK is a downstream effector kinase of $\beta 1$ integrin signaling and plays an imperative role in controlling ECM expression and deposition. To further explore the role of $\beta 1$ integrin recruitment and subsequent activation in the tPA-promoted myofibroblast activation, we examined the effect of inhibiting ILK, the major downstream effector kinase of $\beta 1$ integrin, on $\alpha$-SMA and type I collagen expression induced by tPA and TGF- $\beta 1$. NRK-49F cells were incubated with QLT0267, a selective small molecule inhibitor of $\operatorname{ILK}(25,26)$. As shown in Figure 7, QLT0267 pretreatment completely abrogated the synergistic induction of both $\alpha$-SMA (Figure 7A) and type I collagen (Figure 7, B and C) triggered by $\mathrm{tPA}$ and TGF- $\beta 1$.

We next examined the role of ILK in mediating the fibrogenic action of tPA by disrupting $\beta 1$ integrin/ILK engagement and interaction. It has been known that ILK, via its C-terminal kinase domain, interacts with the cytoplasmic domain of $\beta 1$ integrin $(27,28)$. Therefore, the truncated C-terminal ILK fragment that harbors the integrin binding site would disrupt the $\beta 1$ integrin/ ILK interaction in a dominant-negative fashion. NRK-49F cells were infected with either adenoviral vector that contains the Flagtagged C-terminal ILK fragment (Ad.Flag-C-ILK) (29) or control adenovirus (Ad.LacZ), followed by incubating with tPA in the absence or presence of TGF- $\beta 1$. Expression of the Flag-tagged C-terminal ILK fragment in NRK-49F cells was confirmed by western blot with anti-Flag antibody (Figure 7D). Notably, disruption of $\beta 1$ integrin/ILK interaction by overexpressing a dominant-negative, truncated C-terminal ILK fragment also abrogated the tPAmediated induction of myofibroblast activation. Taken together, both the engagement of $\beta 1$ integrin/ILK and ILK activity are necessary for tPA to promote myofibroblast activation.

Ectopic expression of ILK mimics tPA and synergistically promotes myofibroblast activation. We further investigated the role of ILK activation in mediating the fibrogenic action of tPA by ectopic expression of exogenous ILK. NRK-49F cells were infected with adenovirus harboring ILK or $\beta$-galactosidase gene. As shown in Figure 8A, infection with ILK adenoviral vector caused an increased ILK protein expression in NRK-49F cells, as demonstrated by western blot analysis. We found that exogenous ILK potentiated the TGF- $\beta 1-$ mediated $\alpha$-SMA expression in NRK-49F cells (Figure 8A). Ectopic expression of exogenous ILK also synergistically promoted type I collagen mRNA and protein expression (Figure 8, B-D). Hence ILK mimics tPA and plays a role in mediating the effect of tPA on myofibroblast activation. 
A
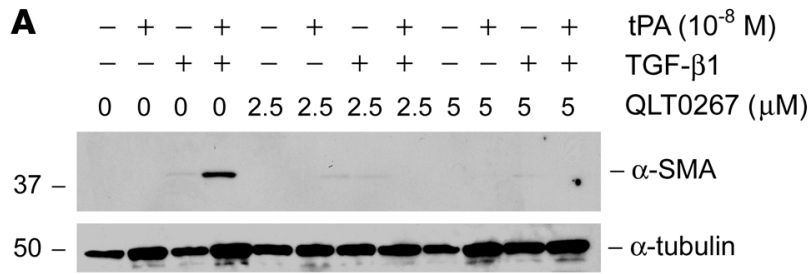

C

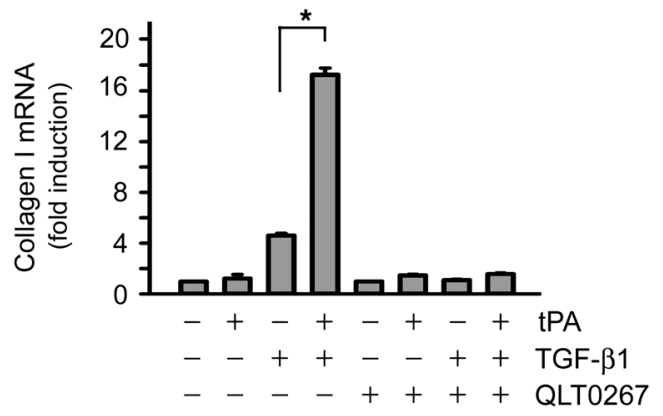

B

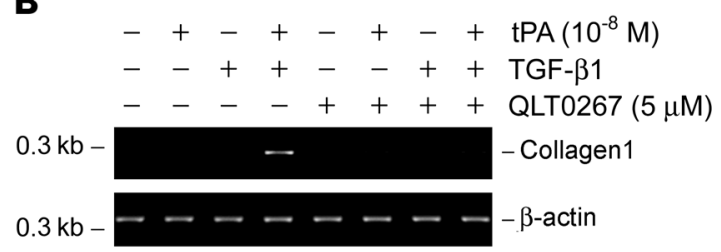

D

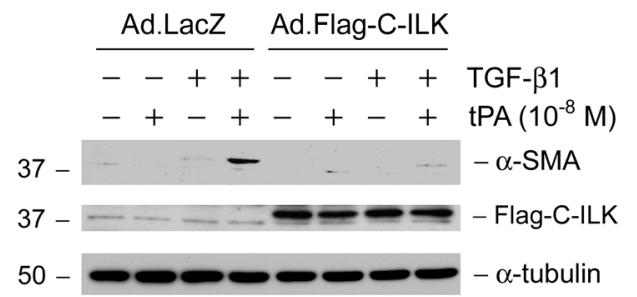

Figure 7

Inhibition of the ILK or disruption of the $\beta 1$ integrin/ILK signaling abrogates the profibrotic effect of tPA. (A-C) NRK-49F cells were pretreated with ILK inhibitor QLT0267 (5 $\mu \mathrm{M})$ for 1 hour and then treated with tPA in the absence or presence of TGF- $\beta 1$ for 24 hours. QLT0267 inhibited the tPA-mediated induction of $\alpha$-SMA (A). QLT0267 also inhibited the tPA-mediated induction of type I collagen in NRK-49F cells (B and C). Quantitative determination of type I collagen mRNA levels is presented in $\mathbf{C}$. ${ }^{*} P<0.05$. (D) Disruption of $\beta 1$ integrin/ILK signaling, by overexpressing a dominant-negative truncated C-terminal ILK fragment that harbors the integrin binding site, also abrogated the tPA-mediated induction of myofibroblast activation. NRK-49F cells were infected with either control adenovirus (Ad.LacZ) or adenoviral vector that contained the Flag-tagged C-terminal ILK fragment (Ad.Flag-C-ILK), followed by incubation with tPA in the absence or presence of TGF- $\beta 1$. Expression of the Flag-tagged C-terminal ILK fragment in NRK-49F cells was confirmed by western blot analysis with anti-Flag antibody.

tPA promotes $L R P-1 / \beta 1$ integrin interaction and myofibroblast activation in vivo. To establish the in vivo relevance of LRP- $1 / \beta 1$ integrin signaling in mediating the fibrogenic action of $\mathrm{tPA}$, we investigated the interaction between LRP- 1 and $\beta 1$ integrin in the obstructed kidney at 7 days after unilateral ureteral obstruction (UUO). Kidney homogenates were immunoprecipitated with anti- $\beta 1$ integrin antibody, followed by immunoblotting with antibodies against LRP-1 and $\beta 1$ integrin, respectively. As shown in Figure 9, $A$ and $B, L R P-1 / \beta 1$ integrin complex was significantly reduced in the obstructed kidney of homozygous tPA knockout $\left(t P A^{-/-}\right)$mice compared with wild-type controls $\left(t P A^{+/+}\right)$. Double-staining demonstrated that tPA and LRP-1 were predominantly localized in renal interstitial cells, and their distribution pattern largely overlapped (Figure 9C). Furthermore, double-staining for $\alpha$-SMA and LRP-1 revealed that LRP-1 was mainly localized in interstitial $\alpha$-SMApositive myofibroblasts in the obstructed kidney (Figure 9D). Consistent with previous reports $(11,15)$, ablation of $\mathrm{tPA}$ reduced $\alpha$-SMA and LRP-1 abundance after obstructive injury. Therefore, it appears clear that tPA may also promote myofibroblast activation via a LRP-1-dependent pathway in vivo.

\section{Discussion}

Myofibroblast activation, as manifested by de novo $\alpha$-SMA expression, is a crucial event that plays a fundamental role in the ECM overproduction and deposition in fibrotic kidney $(1,2)$. The results presented in this study demonstrate that tPA promotes renal interstitial myofibroblast activation and ECM accumulation, consistent with our previous in vivo observation that tPA ablation prevents kidney fibrosis with less myofibroblast and ECM accumulation after obstructive injury (11). The fibrogenic effect of tPA is clearly independent of its protease activity, but it requires mem- brane receptor LRP-1-mediated cellular signaling. As illustrated in Figure 10, tPA activates LRP-1 receptor by inducing its tyrosine phosphorylation, which leads to the recruitment of $\beta 1$ integrin through facilitating LRP- $1 / \beta 1$ integrin interaction and subsequently activates the ILK signaling. Blockade of each and every step within this signaling circuit abolishes the fibrogenic effect of tPA on myofibroblast activation (Figure 10). These studies have established a novel signal pathway in which tPA functions as a fibrogenic cytokine that transmits its signal across plasma membrane to dictate the fibrosis-related gene expression.

tPA is traditionally viewed as a simple serine protease whose main function is to cleave plasminogen into plasmin, a protease that in turn can activate some MMPs from their zymogen to active forms. In addition, tPA also regulates the posttranslational activation of several growth factors such as HGF (directly) or TGF- $\beta 1$ (indirectly via plasmin) through its proteolytic capacity $(19,30)$. As these growth factors are capable of modulating myofibroblast activation in different ways, one potential explanation for tPA promotion of the TGF- $\beta 1$-induced $\alpha$-SMA and type I collagen expression in renal fibroblasts could be an indirect consequence of its protease activity, as it may activate an intermediate mediator that is actually responsible for its profibrotic action. However, 2 experiments employed in this study clearly exclude that possibility. First, inhibition of plasmin activity by aprotinin appears to have no effect on the $\alpha$-SMA induction by tPA, suggesting that tPA action is independent of plasmin generation. Furthermore, the nonenzymatic mutant tPA, which has lost the enzyme catalytic activity due to mutation in the active site of the protease $(20,21)$, still retains its ability to promote myofibroblast activation. This conclusively demonstrates that tPA promotes myofibroblast activation by a mechanism independent of its protease activity. 
A

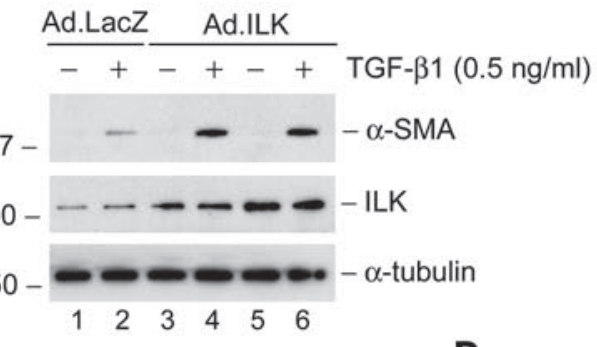

C

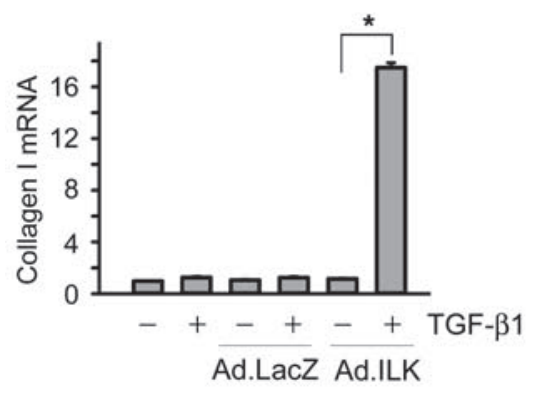

D

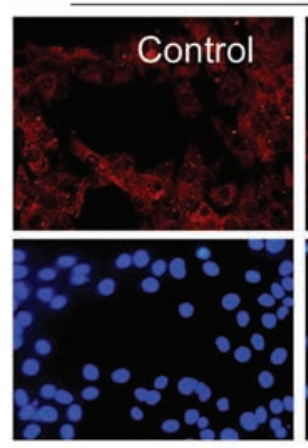

B

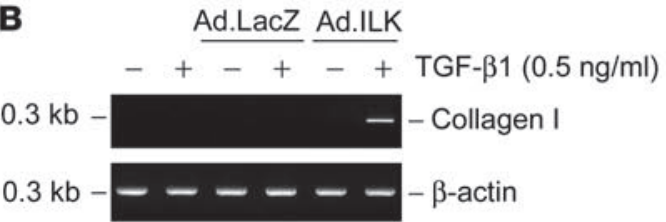

Figure 8

Ectopic expression of ILK mimics tPA and synergistically promotes myofibroblast activation. (A) NRK-49F cells were infected with different dosages of adenovirus harboring ILK gene (Ad.ILK) (lanes 3 and 4, $2 \times 10^{7}$ particle/ml; lanes 5 and $6,4 \times 10^{7}$ particle/ml) or $\beta$-galactosidase (Ad.LacZ) (lanes 1 and $2 ; 4 \times 10^{7}$ particle/ml). Exogenous ILK mimicked tPA and promoted TGF- $\beta 1$-mediated $\alpha$-SMA expression. (B and C) Ectopic expression of exogenous ILK synergistically promoted type I collagen mRNA expression. Quantitative results are presented in C. ${ }^{*} P<0.05$. (D) Immunofluorescence staining showed that exogenous ILK synergistically promoted type I collagen protein expression in NRK-49F cells. Scale bar: $20 \mu \mathrm{m}$.

Instead, tPA action is primarily contingent on its membrane receptor LRP-1-mediated signaling. Our data reinforce the notion that tPA possesses both protease activity and cytokine function, perhaps in a non-mutually exclusive fashion.

The cytokine function of tPA is apparently mediated by its cell membrane receptor LRP-1 (15). It has been shown that tPA specifically binds to LRP-1 in renal fibroblasts, which can be blocked by LRP-1 antagonist RAP (15). More importantly, tPA binding triggers a rapid and robust LRP-1 phosphorylation on tyrosine residues in the $\beta$ subunit, which initiates its downstream signal transduction and MMP-9 gene transactivation (15). Similarly, tPA promotion of myofibroblast activation also requires LRP-1, as inhibition of tPA binding to LRP-1 by RAP abolishes the fibrogenic effects of tPA (Figure 3). Notably, RAP appears also to have a tendency to block the TGF- $\beta 1$-stimulated $\alpha$-SMA expression in NRK-49F cells in the absence of exogenous tPA (Figure 3A). The reason behind this observation is unknown, but it could be attributable to that TGF- $\beta 1$ induces endogenous tPA expression (31). Therefore, under the basal conditions without exogenous tPA, LRP-1 activation by the trivial amount of endogenous tPA may also play a role in synergistically promoting TGF- $\beta 1$-stimulated $\alpha$-SMA expression. Direct evidence for the involvement of LRP-1 is illustrated by the observation that tPA can not promote TGF- $\beta 1$-mediated $\alpha$-SMA expression in the LRP- 1 -deficient fibroblasts (Figure 3). Furthermore, tyrosine phosphorylation of LRP-1 $\beta$ subunit is required for mediating the synergistic effect of tPA on myofibroblast activation. Collectively, these data underscore that activation of the membrane receptor LRP-1 plays an indispensable role in mediating the cytokine function of tPA. It is of interest to note that connective tissue growth factor (CTGF), another ligand of LRP-1, also promotes myofibroblast activation by a mechanism dependent on LRP-1 tyrosine phosphorylation (32). In this regard, tPA shares a close functional similarity to CTGF, a well-known profibrotic cytokine.

One of the novel findings in this study is that tPA promotes the interaction of LRP- 1 with $\beta 1$ integrin, thereby facilitating the LRP-1-mediated recruitment of integrin signaling. The physical connection between LRP- 1 and $\beta 1$ integrin, as shown by coimmunoprecipitation, provides an intrinsic link of tPA/LRP-1 to integrin signaling. Such interaction is apparently operative in vivo, as tPA deficiency reduces the LRP- $1 / \beta 1$ integrin complex formation in the obstructed kidney (Figure 9 ). Integrins are heterodimeric adhesion receptors consisting of noncovalently associated $\alpha$ and $\beta$ subunits. As ECM receptors, integrins mediate bidirectional biochemical signals (inside-out or outside-in signaling) that regulate a wide variety of cellular responses, such as cell survival, proliferation, and matrix deposition $(33,34)$. The $\beta 1$ integrin is particularly interesting because it has been implicated in the pathogenesis of various fibrotic diseases $(35,36)$. In renal interstitial fibroblasts, LRP- 1 and $\beta 1$ integrin appear to constitutively interact under basal conditions (Figure 5), suggesting a potential integration of the signals triggered by these 2 membrane receptor systems. However, tPA markedly promotes the interaction between LRP- 1 and $\beta 1$ integrin, leading to the recruitment of integrin signaling. Increased recruitment of $\beta 1$ integrin will inevitably cause its "clustering," a mode of activation that is well recognized for integrin signaling.

How exactly tPA promotes the physical interaction of LRP-1 and $\beta 1$ integrin in renal fibroblasts remains elusive at this stage. It is clear, however, that short-term treatment of NRK-49F cells 
A

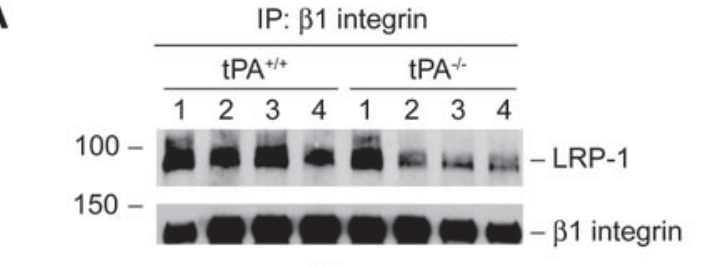

C
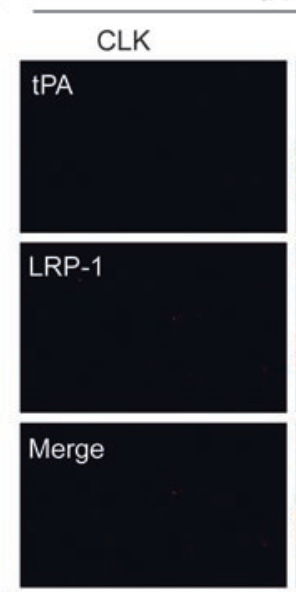

D
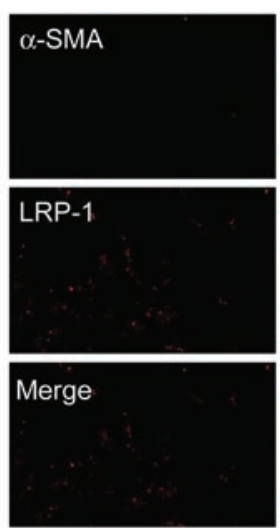

$\mathrm{tPA}^{+/+}$
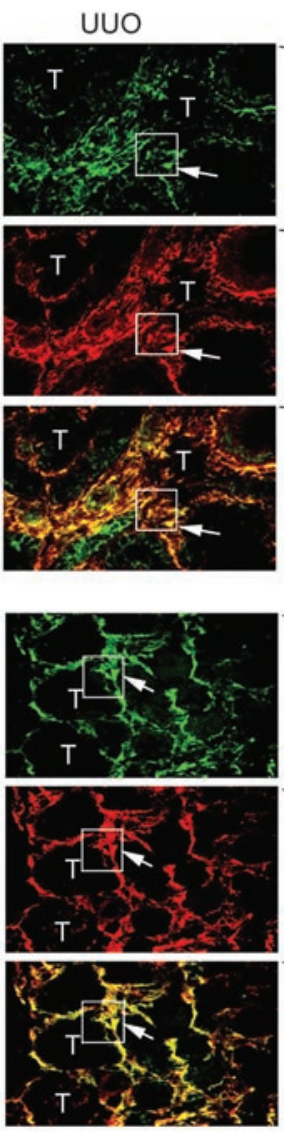
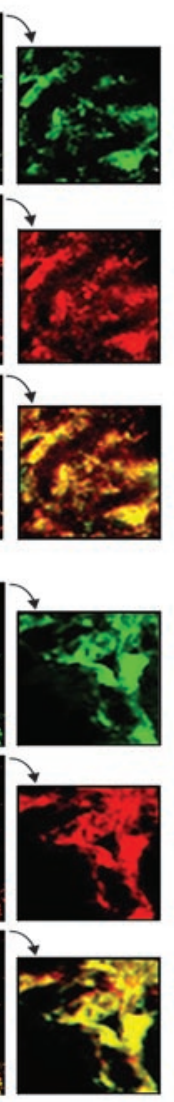
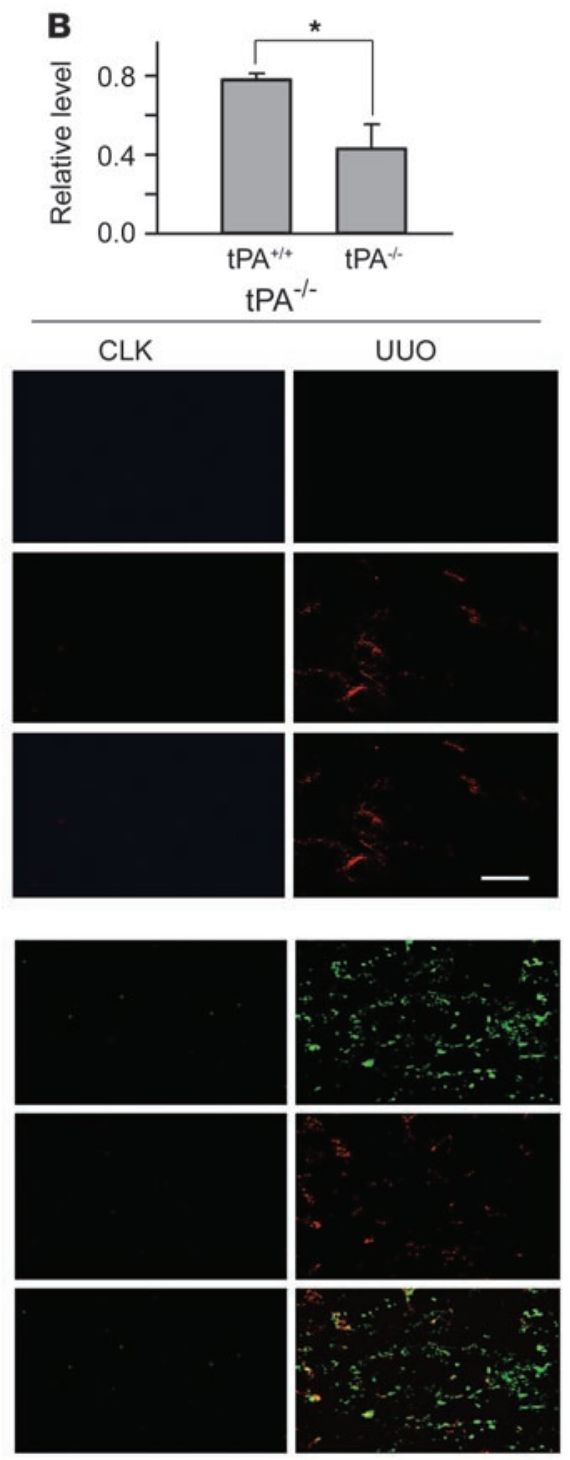

\section{Figure 9}

tPA promotes LRP-1/ $\beta 1$ integrin interaction and myofibroblast activation in obstructive nephropathy. (A and B) Ablation of tPA reduced the LRP- $1 / \beta 1$ integrin complex formation in the obstructed kidney at 7 days after UUO. Kidney homogenates were immunoprecipitated with anti- $\beta 1$ integrin antibody, followed by blotting with antibodies against LRP-1 and $\beta 1$ integrin. Lane numbers coincide with individual animal numbers (A). Quantitative determination of the relative levels of LRP-1/ $\beta 1$ integrin complex is presented in B. ${ }^{*} P<0.05(n=4)$. (C and D) Colocalization of tPA and LRP-1, as well as $\alpha$-SMA and LRP-1, in vivo. Kidney sections from $t P A^{+/+}$and $t P A^{-/}$mice were prepared at 7 days after UUO and double-stained for tPA and LRP-1 (C) or $\alpha$-SMA and LRP-1 (D). Arrows indicate the cells with positive staining. Enlarged images of the areas boxed with white lines are also presented. T, tubular compartment; CLK, control contralateral unobstructed kidneys. Scale bar: $40 \mu \mathrm{m}$. Original magnification, $\times 3.5$ (insets).

with tPA (or RAP and genistein) does not affect total cellular $\beta 1$ integrin abundance. Likewise, tPA also fails to increases cell surface levels of $\beta 1$ integrin by promoting its maturation (37), as demonstrated by a cell membrane surface protein biotinylation assay. Therefore, the increased LRP- $1 / \beta 1$ integrin interaction, which occurs rapidly after tPA stimulation, is likely caused by the LRP-1-mediated recruitment of the preexisting $\beta 1$ integrin in plasma membrane. Such recruitment of $\beta 1$ integrin is dependent on the tPA-triggered tyrosine phosphorylation of the LRP-1 $\beta$ subunit, as inhibition of tyrosine phosphorylation abolishes LRP- $1 / \beta 1$ integrin interaction and myofibroblast activation. It is interesting to note that tPA appears not to bind to $\beta 1$ integrin directly, as demonstrated by coimmunoprecipitation in the LRP-1deficient PEA-13 cells (data not shown). This implies that $\beta 1$ integrin may function as a tPA "coreceptor," but its action absolutely depends on the activation of the primary receptor, LRP-1. Furthermore, although LRP-1 is identical to T $\beta R-V$ and mediates TGF- $\beta$-induced growth inhibition in certain cell types $(23,24)$, depletion of TGF- $\beta$ does not affect tPA-mediated LRP- 1 phosphorylation and its recruitment of $\beta 1$ integrin (Figures $4 \mathrm{~F}$ and $5 \mathrm{G}$ ), suggesting that tPA facilitates LRP- $1 / \beta 1$ integrin interaction by a TGF- $\beta$-independent mechanism. Taken together, we propose that tPA binds to its membrane receptor LRP-1 and induces it to undergo tyrosine phosphorylation, which alters the LRP-1 
Basal condition

tPA stimulation

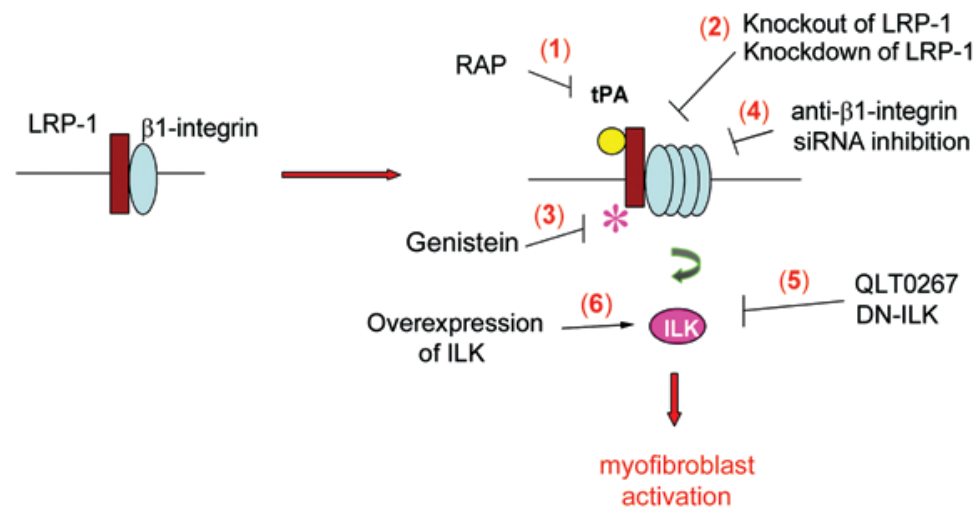

Figure 10

Schematic illustration of the signaling transduction pathway leading to tPA promotion of myofibroblast activation. tPA binds to membrane receptor LRP-1, induces its tyrosine phosphorylation, which leads to the increased recruitment of $\beta 1$ integrin and activates downstream ILK. Blockade of each and every step within this signaling circuit by multiple strategies (numbers 1-5) abolishes the fibrogenic effect of tPA on myofibroblast activation, whereas over-expression of ILK mimics TPA and promotes the TGF- $\beta 1-$ mediated myofibroblast activation (number 6 ).

3 -dimensional structure and renders it able to recruit $\beta 1$ integrin, consequently activating its downstream signaling.

Increased recruitment of $\beta 1$ integrin after tPA stimulation will certainly lead to an activation of its downstream signaling. ILK, an intracellular serine/threonine protein kinase $(27,28)$, is a major downstream effector of $\beta 1$ integrin that is known to be implicated in the pathogenesis of a wide variety of CKDs, including nephrotic syndrome and diabetic and obstructive nephropathy (38-40). ILK, via its $\mathrm{C}$-terminal kinase region, binds to the cytoplasmic domain of $\beta 1$ integrin and mediates its signaling in diverse types of cell. Earlier studies have shown that ILK is a key mediator for the tubular epithelial-mesenchymal transition (40), a phenotypic conversion process that leads to generation of matrix-producing cells in the fibrotic kidney $(41,42)$. Clearly, the $\beta 1$ integrin/ILK pathway is essential for mediating the fibrogenic action of $\mathrm{tPA}$, as blockade of $\beta 1$ integrin signaling (Figure 6), inhibition of ILK activity, or disruption of $\beta 1$ integrin/ILK engagement (Figure 7) completely abrogates the synergistic induction of $\alpha$-SMA and type 1 collagen by tPA. Consistently, ectopic expression of ILK by adenoviral vector in NRK-49F cells mimics tPA action and synergistically promotes TGF- $\beta 1$-mediated myofibroblast activation (Figure 8). Therefore, ILK is not only necessary but also sufficient for mediating the fibrogenic action of tPA.

In summary, we have shown for what we believe to be the first time that tPA promotes TGF- $\beta 1$-mediated renal interstitial myofibroblast activation and ECM production by a mechanism independent of its protease activity. tPA elicits its fibrogenic action by a novel signal transduction pathway in which it binds to the membrane receptor LRP-1 and triggers the receptor tyrosine phosphorylation that in turn recruits $\beta 1$ integrin and subsequently activates ILK signaling. These findings establish that, in addition to its protease activity, tPA functions as a cytokine that is capable of transmitting its signal from the extracellular environment across the plasma membrane to control gene expression. Because
tPA also promotes the TGF- $\beta 1$-mediated activation of the matrix-producing cells from nonrenal tissues such as lung fibroblasts and hepatic stellate cells, our study may have broad implications and could be of general importance in organ fibrogenesis.

\section{Methods}

Antibodies and reagents. The mouse anti- $\alpha$-SMA, anti- $\alpha$-tubulin antibodies, and normal mouse IgG were obtained from Sigma-Aldrich. The anti- $\beta 1$ integrin antibody and specific phospho-tyrosine antibody PY20 were purchased from BD Biosciences - Pharmingen. The antibodies against human tPA and 39-kDa RAP were obtained from Oxford Biomedical Research Inc. Mouse monoclonal anti-LRP-1 (11H4) antibody was described previously (15). Rabbit polyclonal anti-LRP-1 2629 antibody was kindly provided by Dudley Strickland of the University of Maryland (Rockville, Maryland, USA). The rabbit anti- $\beta 1$ integrin antibody used for immunoprecipitation was purchased from Chemicon. Antiphospho-Smad3 was bought from Cell Signaling Technology, and total Smad3 antibody was from Zymed Laboratories. The secondary HRP-conjugated antibodies were obtained from Sigma-Aldrich and Chemicon. Recombinant human singlechain tPA was purchased from American Diagnostica Inc. The non-enzymatic tPA was supplied by Molecular Innovations Inc. The monoclonal pan-specific anti-TGF- $\beta$ neutralizing antibody and recombinant human TGF- $\beta 1$ was obtained from R\&D Systems. Aprotinin, genistein, PD98059, wortmannin, SC68376, and rabbit anti-type I collagen antibody were obtained from Calbiochem-Novabiochem Corp. SB431542 was obtained from Sigma-Aldrich. ILK inhibitor QLT0267 was provided by QLT Inc. $(25,26)$. Streptavidin-agarose bead was purchased from Upstate Biotechnology. Cell culture media, FBS, and supplements were purchased from Invitrogen. All other chemicals were of analytic grade and were obtained from Sigma-Aldrich or Fisher unless otherwise indicated.

Cell culture and treatments. NRK-49F cells, mouse homozygous LRP-deficient embryo fibroblasts (PEA-13), and human lung fibroblasts (MRC-5) were purchased from the American Type Culture Collection. Primary cultured rat hepatic stellate cells were prepared as described previously (43). NRK-49F cells were cultured in Dulbecco's modified Eagle's medium/Ham's F12 (1:1) supplemented with 5\% FBS, while PEA-13, MRC-5, and hepatic stellate cells were incubated in Dulbecco's modified Eagle's medium containing $4 \mathrm{mM}$ L-glutamine, $1.5 \mathrm{~g} / 1$ sodium bicarbonate, $4.5 \mathrm{~g} / 1$ glucose, $1.0 \mathrm{mM}$ sodium pyruvate, and 10\% FBS. Cells were seeded onto 6-well plates and maintained in complete medium until growth to $70 \%$ confluence, then changed to serum-free medium for 24 hours. Recombinant human single-chain tPA was reconstituted according to the manufacturer's instructions (American Diagnostica Inc.) and added to the culture medium at different concentrations for various periods of time as indicated in the figure legends, in the absence or presence of $0.5 \mathrm{ng} / \mathrm{ml} \mathrm{TGF-} \beta 1$ unless otherwise specified. For controls, cells were treated with vehicle. In some experiments, cells were pretreated for 30 minutes with various chemical inhibitors at the concentrations specified in the figure legends, followed by incubation with vehicle, tPA, TGF- $\beta 1$, or tPA plus TGF- $\beta 1$ for an additional 24 hours. For siRNA inhibition, NRK-49F cells were transfected with control siRNA, $\beta 1$ integrin-specific siRNA (Invitrogen) or LRP-1specific siRNA (Dharmacon) at a final concentration of $60 \mathrm{nM}$ by using Lipofectamine 2000 reagent (Invitrogen) as previous described (44). Three days later, cells were treated with tPA and/or TGF- $\beta 1$ for an additional 24 hours and then collected for various assays. 
RT-PCR. Total RNA was extracted using a TRIzoL reagent according to the manufacturer's instructions (Invitrogen) (45). Two $\mu$ g of total RNA was reverse transcribed into cDNA in $20 \mu \mathrm{l}$ reaction buffer by AMV reverse transcriptase (Promega) using random primers at $42^{\circ} \mathrm{C}$ for 30 minutes. PCR was performed for 35 cycles of amplification as follows: $94^{\circ} \mathrm{C}$ for 1 minute, $55^{\circ} \mathrm{C}$ for 1 minute, and $72^{\circ} \mathrm{C}$ for 1 minute, followed by a final extension step at $72^{\circ} \mathrm{C}$ for 10 minutes using HotStar Taq polymerase (Qiagen Inc.) and specific primers designed for rat or mouse collagen $\alpha 1$ (I), $\beta$-actin, rat $\alpha$-SMA, and fibronectin. PCR products were size fractionated on $1.0 \%$ agarose gel and detected by ethidium bromide staining. No detectable signal was found in a parallel control tube without reverse transcriptase or cDNA. The relative abundance of mRNA was determined by the intensity ratio of collagen $\alpha 1$ (I) to $\beta$-actin. The sequences of primer pairs were reported elsewhere (46), except for mouse collagen $\alpha 1$ (I), which was as follows: forward, 5'-ATCTCCTGGTGCTGATGGAC; reverse, 5'ACCTTGTTTGCCAGGTTCAC.

Western blot analysis. Whole-cell lysates were prepared as previously described (47). Samples were heated at $100^{\circ} \mathrm{C}$ for about 5-10 minutes before loading on $10 \%$ SDS polyacrylamide gels. After the proteins were electrotransferred to a PVDF membrane (Amersham Biosciences), nonspecific binding to the membrane was blocked for 1 hour at room temperature with $5 \%$ Carnation nonfat milk in TBST buffer $(20 \mathrm{mM}$ Tris- $\mathrm{HCl}$, $150 \mathrm{mM} \mathrm{NaCl}$, and $0.1 \%$ Tween 20). The membranes were then incubated overnight at $4{ }^{\circ} \mathrm{C}$ with various primary antibodies in blocking buffer at the dilution recommended by the manufacturers. After being extensively washed in TBST buffer, the membranes were incubated with HRP-conjugated secondary antibodies for 1 hour in TBST buffer with 5\% nonfat milk. Membranes were washed with TBST buffer, and the signals were visualized using the SuperSignal West Pico Chemiluminescent Substrate kit (Pierce Biotechnology).

Zymographic analysis. Zymographic analysis of plasmin proteolytic activity was performed according to the method described previously (11). Briefly, tPA was incubated with excess plasminogen at $37^{\circ} \mathrm{C}$ to cleave plasminogen into plasmin. Aliquots of the resultant plasmin/tPA mixture containing approximately $0.25 \times 10^{-8}$ to $1.0 \times 10^{-8} \mathrm{M}$ tPA were loaded into 2 identical $10 \%$ SDS-polyacrylamide gels containing $10 \mathrm{mg} / \mathrm{ml}$ casein (Bio-Rad). After electrophoresis, SDS was removed from the gels by incubation in $2.5 \%$ Triton X-100 at room temperature for 30 minutes with gentle shaking. The gels were washed well with distilled water and incubated at $37^{\circ} \mathrm{C}$ overnight in a developing buffer in the absence or presence of aprotinin $(50 \mathrm{U} / \mathrm{ml})$. The gels were then stained with a solution of $30 \%$ methanol, $10 \%$ glacial acetic acid, and $0.5 \%$ Coomassie blue G250 followed by destaining in the same solution without dye. Proteinase activity was detected as unstained bands on a blue background representing areas of casein digestion.

Coimmunoprecipitation. For the detection of tyrosine phosphorylation on the LRP-1 $\beta$ subunit, NRK-49F cells were treated with $10^{-8} \mathrm{M}$ tPA for various periods of time, then subjected to immunoprecipitation, as described previously (15). Some cells were pretreated with $50 \mu \mathrm{M}$ genistein for 1 hour and then subjected to similar tPA incubation. Cells were lysed in CHAPS buffer (10 mM CHAPS, 20 mM HEPES, pH 7.4, $150 \mathrm{mM}$ $\mathrm{NaCl}, 2 \mathrm{mM} \mathrm{CaCl}_{2}$ ) supplemented with phosphatase and protease inhibitor cocktails (Sigma-Aldrich). The resulting lysates were incubated with the monoclonal antibody against LRP-1 $(11 \mathrm{H} 4)$ at $4{ }^{\circ} \mathrm{C}$ overnight. The protein A/G PLUS agarose beads (Santa Cruz Biotechnology Inc.) were added and incubated at $4^{\circ} \mathrm{C}$ for 3 hours. The beads were then washed with CHAPS buffer, and the proteins were extracted in reducing sampling buffer, separated on $10 \%$ SDS polyacrylamide gel, and analyzed by western blotting using anti-phosphotyrosine and anti-LRP-1 antibodies. For detecting the interaction between LRP- 1 and $\beta 1$ integrin, cell lysates were immunoprecipitated by either anti-LRP- 1 or anti- $\beta 1$ integrin antibodies, followed by immunoblotting with anti- $\beta 1$ integrin or anti-LRP-1 antibodies, respectively.

Indirect immunofluorescence staining. Cells cultured on coverslips were fixed in PBS containing 3\% paraformaldehyde and $0.2 \%$ Triton X-100 for 10 minutes at room temperature (47). After being blocked with $10 \%$ normal donkey serum in PBS for 30 minutes, cells were incubated with primary anti-collagen I antibody at $4{ }^{\circ} \mathrm{C}$ overnight, followed by incubation with cyanine 2- (CY2-) or CY3-conjugated affinity-purified secondary antibody (Jackson ImmunoResearch Laboratories Inc.). Stained cells were mounted with Vectashield anti-fade mounting media with DAPI (4', 6-diamidino2-phenylindole, $\mathrm{HCl}$ ) to visualize nuclei (Vector Laboratories Inc.) and viewed under an Eclipse E600 epifluorescence microscope equipped with a digital camera (Nikon Inc.). For double-staining, kidney cryosections were prepared at 5- $\mu \mathrm{m}$ thickness, fixed, and blocked as described above. Sections were stained with primary anti-tPA or anti- $\alpha$-SMA antibody, followed by staining with CY2-conjugated secondary antibody. Sections were then blocked with goat anti-rabbit IgG Fab fragment overnight and subsequently stained with primary anti-LRP-1 2629 and CY3-conjugated secondary antibody. Sections stained without primary antibodies served as negative controls.

Cell membrane surface protein biotinylation. Cell membrane protein biotinylation assay was performed using water-soluble membrane impermeable EZ-Link sulfo-NHS-LC-biotin reagent, according to the manufacturer's instructions (Pierce Biotechnology). Briefly, NRK-49F cells were pretreated with $50 \mu \mathrm{M}$ genistein or $0.5 \mu \mathrm{M}$ RAP for 1 hour, followed by incubation with $10^{-7} \mathrm{M}$ tPA for an additional 2 hours. Cells were then extensively washed with ice-cold PBS and incubated with $0.5 \mathrm{mg} / \mathrm{ml}$ EZ-Link sulfo-NHS-LC-biotin in PBS at $4^{\circ} \mathrm{C}$ for 30 minutes. Biotinylation reaction was quenched by washing 3 times with PBS containing $100 \mathrm{mM}$ glycine. After cells were lysed in CHAPS buffer, the biotinylated cell membrane protein was pulled down by incubating with Streptavidin-agarose bead (Upstate) at $4^{\circ} \mathrm{C}$ overnight. The proteins were extracted from the beads in sampling buffer and analyzed by western blotting using anti- $\beta 1$ integrin antibody.

Adenovirus infection. Adenovirus infection of NRK-49F cells was carried out as described previously (15). Briefly, NRK-49F cells were seeded on 6 -well culture plates to $90 \%$ confluence in complete medium. After an overnight incubation, the cultures were changed to serum-free medium. The recombinant adenovirus that harbors the wild-type ILK gene (Ad.ILK), the adenovirus that harbors the C-terminal truncated ILK fragment (Ad.C-ILK) (29), or the control adenovirus containing the $\beta$-galactosidase gene (Ad.LacZ) was added to the cultures at a concentration of $2 \times 10^{7}$ or $4 \times 10^{7}$ particles per $\mathrm{ml}$. Cells were incubated for 24 hours in the absence or presence of TGF- $\beta 1(0.5 \mathrm{ng} / \mathrm{ml})$ and then subjected to western blot analysis, RT-PCR, and immunofluorescence staining.

Animal model. Homozygous tPA knockout $\left(t P A^{-/-}\right)$and wild-type $\left(t P A^{+/+}\right)$mice were generated from heterozygous crosses using descendents from original breeding pairs obtained from P. Carmeliet at the University of Leuven (Leuven, Belgium). Animal studies were performed using an approved protocol by the Institutional Animal Care and Use Committee at the University of Pittsburgh. Genotype was confirmed by using PCR amplification of genomic DNA from tail snips as previously described (11). The left kidneys of sex-matched mice weighing 20-22 g (4 animals per group) were subjected to UUO using established procedures described elsewhere (11). The right unobstructed kidneys served as controls. At day 7 after UUO, mice were sacrificed and kidney cryosections were used for immunofluorescence staining. Kidney homogenates were subjected to immunoprecipitation for assessing the interaction of LRP- 1 and $\beta 1$ integrin in vivo. 
Statistics. Statistical analysis of the data was performed using SigmaStat software (Jandel Scientific Software). Comparison between multiple groups was made using 1-way ANOVA followed by the Student-Newman-Keuls test or Student's $t$ test when comparing differences between 2 groups. A $P$ value of less than 0.05 was considered significant.

\section{Acknowledgments}

We thank Yu Yang and Xiaoyue Tan for excellent technical assistance. This work was supported by the NIH grants DK061408, DK064005, and DK071040 (to Y. Liu) and DK054639 (to C. Wu).

1. Liu, Y. 2006. Renal fibrosis: New insights into the pathogenesis and therapeutics. Kidney Int. 69:213-217.

2. Eddy, A.A. 2000. Molecular basis of renal fibrosis. Pediatr. Nephrol. 15:290-301.

3. Strutz, F., and Zeisberg, M. 2006. Renal fibroblasts and myofibroblasts in chronic kidney disease. J. Am. Soc. Nephrol. 17:2992-2998.

4. Neilson, E.G. 2006. Mechanisms of disease: Fibroblasts - a new look at an old problem. Nat. Clin. Pract. Nephrol. 2:101-108.

5. Boukhalfa, G., Desmouliere, A., Rondeau, E. Gabbiani, G., and Sraer, J.D. 1996. Relationship between alpha-smooth muscle actin expression and fibrotic changes in human kidney. Exp. Nephrol. 4:241-247.

6. Muchaneta-Kubara, E.C., and el Nahas, A.M. 1997. Myofibroblast phenotypes expression in experimental renal scarring. Nephrol. Dial. Transplant. 12:904-915.

7. Roberts, I.S., Burrows, C., Shanks, J.H., Venning, M., and McWilliam, L.J. 1997. Interstitial myofibroblasts: predictors of progression in membranous nephropathy. J. Clin. Pathol. 50:123-127.

8. Badid, C., Vincent, M., Fouque, D., Laville, M., and Desmouliere, A. 2001. Myofibroblast: a prognostic marker and target cell in progressive renal disease. Ren. Fail. 23:543-549.

9. Yang, J., and Liu, Y. 2001. Dissection of key events in tubular epithelial to myofibroblast transition and its implications in renal interstitial fibrosis. Am. J. Pathol. 159:1465-1475.

10. Iwano, M., et al. 2002. Evidence that fibroblasts derive from epithelium during tissue fibrosis. J. Clin. Invest. 110:341-350. doi:10.1172/JCI200215518.

11. Yang, J., et al. 2002. Disruption of tissue-type plasminogen activator gene in mice reduces renal interstitial fibrosis in obstructive nephropathy. J. Clin. Invest. 110:1525-1538. doi:10.1172/ JCI200216219.

12. Nassar, T., et al. 2004. In vitro and in vivo effects of tPA and PAI-1 on blood vessel tone. Blood. 103:897-902.

13. Yepes, M., et al. 2003. Tissue-type plasminogen activator induces opening of the blood-brain barrier via the LDL receptor-related protein. J. Clin. Invest. 112:1533-1540. doi:10.1172/JCI200319212.

14. Nagai, N., et al. 2001. Tissue-type plasminogen activator is involved in the process of neuronal death induced by oxygen-glucose deprivation in culture. J. Cereb. Blood Flow Metab. 21:631-634.

15. Hu, K., et al. 2006. Tissue-type plasminogen activator acts as a cytokine that triggers intracellular signal transduction and induces matrix metalloproteinase-9 gene expression. J. Biol. Chem. 281:2120-2127.

16. Herz, J., and Strickland, D.K. 2001. LRP: a multifunctional scavenger and signaling receptor. J. Clin. Invest. 108:779-784. doi:10.1172/JCI200113992.

17. Hussain, M.M. 2001. Structural, biochemical and signaling properties of the low-density lipoprotein receptor gene family. Front. Biosci. 6:D417-D428.

18. Strickland, D.K., and Ranganathan, S. 2003. Diverse role of LDL receptor-related protein in the
Kebin $\mathrm{Hu}$ was supported by a postdoctoral fellowship from the American Heart Association Pennsylvania-Delaware Affiliate.

Received for publication April 3, 2007, and accepted in revised form September 19, 2007.

Address correspondence to: Youhua Liu, Department of Pathology, University of Pittsburgh, S-405 Biomedical Science Tower, 200 Lothrop Street, Pittsburgh, Pennsylvania 15261, USA. Phone: (412) 648-8253; Fax: (412) 648-1916; E-mail: liuy@upmc.edu. clearance of proteases and in signaling. J. Thromb. Haemost. 1:1663-1670.

19. Yee, J.A., Yan, L., Dominguez, J.C., Allan, E.H., and Martin, T.J. 1993. Plasminogen-dependent activation of latent transforming growth factor beta (TGF beta) by growing cultures of osteoblast-like cells. J. Cell. Physiol. 157:528-534.

20. Werner, F., Razzaq, T.M., and Ellis, V. 1999. Tissue plasminogen activator binds to human vascular smooth muscle cells by a novel mechanism. Evidence for a reciprocal linkage between inhibition of catalytic activity and cellular binding. J. Biol. Chem. 274:21555-21561.

21. Olson, S.T., et al. 2001. Resolution of Michaelis complex, acylation, and conformational change steps in the reactions of the serpin, plasminogen activator inhibitor-1, with tissue plasminogen activator and trypsin. Biochemistry. 40:11742-11756.

22. Willnow, T.E., Armstrong, S.A., Hammer, R.E., and Herz, J. 1995. Functional expression of low density lipoprotein receptor-related protein is controlled by receptor-associated protein in vivo. Proc. Natl. Acad. Sci. U. S. A. 92:4537-4541.

23. Tseng, W.F., Huang, S.S., and Huang, J.S. 2004. LRP-1/TbetaR-V mediates TGF-beta1-induced growth inhibition in CHO cells. FEBS Lett. 562:71-78.

24. Huang, S.S., et al. 2003. Cellular growth inhibition by IGFBP-3 and TGF-beta1 requires LRP-1. FASEBJ. 17:2068-2081.

25. Younes, M.N., et al. 2005. Integrin-linked kinase is a potential therapeutic target for anaplastic thyroid cancer. Mol. Cancer Ther. 4:1146-1156.

26. Koul, D., et al. 2005. Targeting integrin-linked kinase inhibits Akt signaling pathways and decreases tumor progression of human glioblastoma. $\mathrm{Mol}$. Cancer Ther. 4:1681-1688.

27. Legate, K.R., Montanez, E., Kudlacek, O., and Fassler, R. 2006. ILK, PINCH and parvin: the tIPP of integrin signalling. Nat. Rev. Mol. Cell Biol. 7:20-31.

28. Wu, C. 2005. PINCH, N(i)ck and the ILK: network wiring at cell-matrix adhesions. Trends Cell Biol. 15:460-466.

29. Guo, L., and Wu, C. 2002. Regulation of fibronectin matrix deposition and cell proliferation by the PINCH-ILK-CH-ILKBP complex. FASEB J. 16:1298-1300.

30. Mars, W.M., Zarnegar, R., and Michalopoulos, G.K. 1993. Activation of hepatocyte growth factor by the plasminogen activators uPA and tPA. Am. J. Pathol. 143:949-958.

31. Schacke, W., Beck, K.F., Pfeilschifter, J., Koch, F., and Hattenbach, L.O. 2002. Modulation of tissue plasminogen activator and plasminogen activator inhibitor- 1 by transforming growth factor-beta in human retinal glial cells. Invest. Ophthalmol. Vis. Sci. 43:2799-2805

32. Yang, M., Huang, H., Li, J., Li, D., and Wang, H. 2004. Tyrosine phosphorylation of the LDL receptor-related protein (LRP) and activation of the ERK pathway are required for connective tissue growth factor to potentiate myofibroblast differentiation. FASEB J. 18:1920-1921.
33. Takagi, J., and Springer, T.A. 2002. Integrin activation and structural rearrangement. Immunol. Rev. 186:141-163.

34. Humphries, M.J., Travis, M.A., Clark, K., and Mould, A.P. 2004. Mechanisms of integration of cells and extracellular matrices by integrins. Biochem. Soc. Trans. 32:822-825.

35. Cosgrove, D., et al. 2000. Integrin alpha1beta1 and transforming growth factor-beta 1 play distinct roles in alport glomerular pathogenesis and serve as dual targets for metabolic therapy. Am. J. Pathol. 157:1649-1659.

36. Wagrowska-Danilewicz, M., and Danilewicz, M. 2004. Expression of alpha5beta 1 and alpha6beta 1 integrins in $\operatorname{IgA}$ nephropathy (IgAN) with mild and severe proteinuria. An immunohistochemical study. Int. Urol. Nephrol. 36:81-87.

37. Salicioni, A.M., Gaultier, A., Brownlee, C., Cheezum, M.K., and Gonias, S.L. 2004. Low density lipoprotein receptor-related protein-1 promotes beta1 integrin maturation and transport to the cell surface. J. Biol. Chem. 279:10005-10012.

38. Kretzler, M., et al. 2001. Integrin-linked kinase as a candidate downstream effector in proteinuria. FASEB J. 15:1843-1845.

39. Guo, L., Sanders, P.W., Woods, A., and Wu, C. 2001. The distribution and regulation of integrin-linked kinase in normal and diabetic kidneys. Am. J. Pathol. 159:1735-1742.

40. Li, Y., Yang, J., Dai, C., Wu, C., and Liu, Y. 2003. Role for integrin-linked kinase in mediating tubular epithelial to mesenchymal transition and renal interstitial fibrogenesis. J. Clin. Invest. 112:503-516. doi:10.1172/JCI200317913.

41. Liu, Y. 2004. Epithelial to mesenchymal transition in renal fibrogenesis: pathologic significance, molecular mechanism, and therapeutic intervention. J. Am. Soc. Nephrol. 15:1-12.

42. Kalluri, R., and Neilson, E.G. 2003. Epithelialmesenchymal transition and its implications for fibrosis. J. Clin. Invest. 112:1776-1784. doi:10.1172/ JCI200320530.

43. Riccalton-Banks, L., Bhandari, R., Fry, J., and Shakesheff, K.M. 2003. A simple method for the simultaneous isolation of stellate cells and hepatocytes from rat liver tissue. Mol. Cell. Biochem. 248:97-102.

44. Tan, R., et al. 2006. Downregulation of SnoN expression in obstructive nephropathy is mediated by an enhanced ubiquitin-dependent degradation. J. Am. Soc. Nephrol. 17:2781-2791.

45. Li, Y., et al. 2006. Hepatocyte growth factor is a downstream effector that mediates the antifibrotic action of peroxisome proliferator-activated receptor-\{gamma\} agonists. J. Am. Soc. Nephrol. 17:54-65.

46. Li, Y., Spataro, B.C., Yang, J., Dai, C., and Liu, Y. 2005. 1,25-dihydroxyvitamin D inhibits renal interstitial myofibroblast activation by inducing hepatocyte growth factor expression. Kidney Int. 68:1500-1510.

47. Yang, J., and Liu, Y. 2002. Blockage of tubular epithelial to myofibroblast transition by hepatocyte growth factor prevents renal interstitial fibrosis. J. Am. Soc. Nephrol. 13:96-107. 